\section{Diagnosis and antimicrobial treatment of bacterial Neisseria gonorrhea infections: Update review article}

\section{Abstract}

Sexually transmitted infections (STIS) are caused by a wide spectrum of bacteria, viruses and parasites. These agents can be easily transmitted by direct genital or oral sexual contact. Recently, the World Health Organization (WHO), reported that more than 1 million STIs are acquired every day worldwide. Each year, there are an estimated 357 million new infections with one of the following four STIs: chlamydia, gonorrhea, syphilis and trichomoniasis. There are few studies and official reports published on the prevalence of STIs in most Arab countries. However, a few recent studies showed indicate an increased prevalence of certain STIs in some Arab countries, and few data is available on their antimicrobial susceptibility.
Asem A. Shehabi', Monzer Hamze ${ }^{2}$

\author{
1 Department of Pathology-Microbiology, \\ School of Medicine, The Jordan \\ University, Amman, Jordan \\ 2 Laboratoire Microbiologie Santé et \\ Environnement (LMSE), Ecole Doctorale \\ des Sciences et de Technologie, \\ Faculté de Santé Publique, Université \\ Libanaise,Tripoli, Lebanon
}

Corresponding authors:

\section{Asem A. Shehabi}

"Dasashehabi2@gmail.com

\section{Monzer Hamze}

झםmhamze@monzerhamze.com

\section{Introduction}

Gonorrhea is the second most prevalent bacterial sexually transmitted infection (STI) with a global estimated incidence of 78 million cases annually [1,2], with probably a large number of cases that remain unreported [2]. In the United States of America, N. gonorrhea is the second most commonly reported communicable disease, with more than 350,000 cases reported annually [3]. A recent study from Saudi Arabia reported that nongonococcal urethritis, trichomoniasis, and HIV were the most commonly reported STIs in the Kingdom of Saudi Arabia over the period 2005-2012, and the study showed that gonococcal urethritis accounted for $4.4 \%$ of the total STI cases [4].

Obligate pathogenic organisms of STIs such as gonorrhea and syphilis can be recognized clinically and confirmed by rapid laboratory test in men and women. Whereas, other potential bacterial pathogens of STIs represented by Chlamydia trachomatis, Mycoplasma genitalium and Ureaplasma urealyticum are among the most prevalent agents found in the genital tract of humans [5-6]. These organisms are more difficult to detect and to prove their presence the actual cause of genital infections, since they can contribute to asymptomatic and symptomatic clinical diseases alone or in association with $N$. 
gonorrhea [5-7]. Prompt and accurate treatment of STIs is essential measurement to cure patients and prevents their complications . In addition, rapid detection of positive cases of STIs will help to control and prevent the spread of their harmful agents in the communities. It is important to mention that there is no available vaccine against any type of STIs.

\section{Clinical manifestations of $\boldsymbol{N}$. gonorrhea infections}

The majority of urethral infections caused by $N$. gonorrhea among men produce symptoms that require medical examination [8]. Among women, gonorrhea does not produce recognizable symptoms until complications occur [9]. Because gonococcal infections among women are frequently asymptomatic, the Centers for Disease Control and Prevention (CDC/USA) recommended that clinicians screen all sexually active women, including those who are pregnant, for gonorrhea infection if they are at any increased risk [10]. In general, risk factors of gonorrhea infections include age younger than 25 years, a previous gonorrhea infection, other STIS, and new or multiple sexual partners [8-10].

In Western countries, 10 to $30 \%$ of patients with gonorrhea will have a concomitant Chlamydia infection [6,11-12]. The disease is associated with high morbidity and socioeconomic consequences and remains a public health problem worldwide. N. gonorrhea can also infect other mucosal surfaces, including the pharynx, rectum and conjunctiva, but may rarely invade the bloodstream, causing disseminated infection $[8,11,13]$. In women, the primary gonococcal infection is in the endocervix, with concomitant urethral infection, and it is frequently asymptomatic, but if the infection causes symptoms, the most common is purulent vaginal discharge, which is a result of endocervicitis [8,10-11]. Gonorrhea infections do not cause vaginitis, but other concomitant infections may produce vaginal findings [8]. Ascending infection may occur in 10-20\% of infected women and can result in acute pelvic inflammatory disease (PID) or salpingitis that may manifest as endometritis, and tubo-ovarian abscess, all of which can lead to scarring, ectopic pregnancy, infertility, and chronic pelvic pain [8,13-14]. In men, gonococcus is known to cause urethritis, which is rarely complicated by infections of other parts of the genital tract $[11,13]$. Unlike women, men with urogenital infections are usually symptomatic. The normal incubation period is two to six days after exposure, and symptoms include purulent penile discharge and dysuria. The possibility always remains of untreated gonorrhea in men leading to prostatitis, epididymitis, epididymoorchitis, urethral stricture, and infertility [14,15-16]. Rectal infection with $N$. gonorrhoeae may occur through direct inoculation by receptive anal intercourse and is typically seen in homosexual males but can also appear in women. In addition, patients often do not experience symptoms (two thirds of patients), except for possible occurrences of anal pruritus, painless rectal discharge, and tenesmus. Pharyngeal infections caused by $N$. gonorrhoeae usually occur after orogenital exposure; symptoms are mild or absent $[11,13]$. Adult ocular gonococcal infection is very rare, with transmission usually being by autoinoculation or direct inoculation from the genital secretions of an infected partner [17]. Disseminated infection is rare but it can occur in 1 to $3 \%$ of adults who have gonorrhea. It can occur in both sexes with higher frequency in women [8,11-12 ]. Symptoms of disseminated infection can range from slight joint pain, a few skin lesions, and no fever to overt polyarthritis and a high fever [1112]. Disseminated gonorrhea may also present as bacterial endocarditis and meningitis; yet, the incidences of these presentations have declined with the introducing of antibiotic therapy [11]. During the neonatal period and the first year of life, gonorrhea infections can cause neonatal conjunctivitis (ophthalmia neonatorum), pharyngitis, rectal infections, and, in rare cases, pneumonia $[8,11]$. Preadolescent children most commonly contract gonococcal infec- 
tions through sexual abuse [8]. Rapid diagnosis and administration of antimicrobial therapy are the main measurement to reduce and control of gonorrhea infection in the community and prevent its complications in infected persons $[3,18]$.

\section{Laboratory Diagnosis}

\section{Specimen collection}

The specimen collection method depends on the testing technique used in a laboratory and the age, sex and sexual orientation of the patient. Specimens should be collected with dacron or rayon swabs because calcium alginate may be toxic to gonococci. Fatty acids inhibit the growth of gonococci; therefore, cotton swabs that do not list acceptable manufacturer specifications should not be used [19]. To minimize the inhibitory effects of unknown substances in the specimen, the swabs should be inoculated directly onto growth medium or placed in swab transport medium immediately after sampling [20].

\section{Gram-stain and culture}

Until the late 1980s, laboratory diagnosis of gonorrhea was limited to gram stain and bacterial isolation. Gram staining may be performed directly on exudates from several sites, such as the male urethra, cervix, rectum, synovial fluid, eye discharge, vagina in prepubertal girls, skin lesions, and cerebrospinal fluid. The presence of intracellular gram- negative kidney-shaped diplococci in polymorphonuclear leukocytes on microscopy suggests the diagnosis of a gonococcal infection $[8,12]$. The gram stain is a rapid tool and has comparable sensitivity to bacterial culture for symptomatic urethral gonorrhea in men. However, it is relatively insensitive for specimens collected from women and from extragenital sites where the specificity of gram stain may also be affected by the presence of commensal Neisseria species [20]. The primary specimens should be inoculated onto nonselective chocolate agar and selective agar (Thayer-Martin) containing antimicro- bial agents that inhibit the growth of commensal bacteria and fungi. The inoculated plates should be incubated at $35^{\circ} \mathrm{C}$ to $37^{\circ} \mathrm{C}$ in a moist atmosphere enriched with $\mathrm{CO} 2$ (3\% to 7\%)[19]. After $18 \mathrm{~h}$ to 24 hours culture should be used as the inoculum for additional tests. Plates should not be incubated for longer than $48 \mathrm{~h}$ because most old cultures would not survive storage conditions. Autolysis may occur during prolonged incubation, and growth from agar plates becomes difficult to suspend in solutions.

\section{Confirmation of isolates}

Several Neisseria and related species may be misidentified as $N$. gonorrhea unless appropriate differential tests are performed, especially in specimens obtained for direct smear or isolates from endocervical secretions of women. Therefore, it is essential to confirm $N$. gonorrhea isolates by biochemical tests: $N$ gonorrhea can be differentiated from other Neisseria species, Moraxella species, Kingella species and other commensals based on its ability to grow on appropriate selective and nonselective media, produce acid from glucose, maltose,

lactose, sucrose and fructose, reduce nitrate, produce polysaccharide from sucrose and exhibit DNase production. The Rapid Identification Method-Neisseria (Remel Inc, USA) is a commercially available rapid acid production test that compares well with the conventional method, but may also not differentiate between $N$ gonorrhea and $N$ cinerea [21].

\section{Chromogenic enzyme substrates}

These tests are based on the presence of preformed chromogenic enzyme in the culture and, thus, require a heavy inoculum of the organism grown on selective medium to permit rapid speciation of isolates.

Evaluation of eight methods for identification of pathogenic Neisseria species: Neisseria-Kwik, RIMN, Gonobio-Test, Minitek, Gonochek II, GonoGen, Phadebact Monoclonal GC OMNI Test, and Syva MicroTrak Test [21]. 


\section{Coagglutination tests}

Coagglutination tests can be performe on primary culture and, therefore, confirmed results can be obtained one day earlier than tests that require subculturing from primary culture plates, by using molecular techniques [22], or the Maldi Tof technique [23]. There is no specific and sensitive serological test available to detect recent gonococcal infections through the demonstration of $N$. gonorrhea-specific antibodies or antigens in patients' sera [19].

\section{Molecular detection Methods}

Over the last 20 years, nucleic acid amplification methods (NAATs) have gradually replaced traditional methods for the detection of sexually transmitted infections. NAAT technology comes with some important gains for diagnosis, increased sensitivity, and rapid result for screening of symptomatic and asymptomatic individuals using various clinical specimens [19]. However, NAAT tests have some technical problems. False-negative and false-positive results have been reported using various tests. Additionally, NAAT methods can facilitate detection of C. trachomatis, N. gonorrhoeae, M. genitalium and T. vaginalis [24]. Single detection systems or dual detection tests for C. trachomatis and $N$. gonorrhea are now commercially available The hybridization assays include the Gen- Probe PACE ॥ (Gen-Probe, USA) and the Digene Hybrid Capture II assays (Digene Corp., USA) [25]. These assays use a specific oligonucleotide probe to hybridize directly to $N$. gonorrhea nucleic acid present within a specimen. Reported sensitivity and specificity values of the hybridization assays showed that these may be below that of bacterial culture [25]. Several commercial $N$. gonorrhea NAATs are available since 10 years, and make use of different technologies: Polymerase Chain Reaction (PCR) (Roche Diagnostics, USA), Transcription-Mediated Amplification (TMA) (Gen-Probe, USA), Strand Displacement Amplifica- tion (SDA) (Becton Dickinson, USA), and the Ligase Chain Reaction (LCR) (Abbott Laboratories, USA) [12]. Each of these assays has used a unique $N$. gonorrhea gene target [25]. Multiplex qualitative real-time PCR assay that detects $C$. trachomatis and N. gonorrhea and contains an internal control has been developed. A real-time PCR assay has the advantage of reducing the detection time of regular PCR procedures [24]. In addition to the commercial assays, numerous in house $N$. gonorrhoeae NAAT assays have also been described. Gene targets for in-house PCR assays have included the ORF1, CppB, OMPIII, Opa genes as well as the porA pseudogene [24].

There are several advantages of $N$. gonorrhea NAATs; first, they offer improved sensitivity compared with bacterial culture. The increased sensitivity of NAATs makes them particularly suitable for screening, enabling accurate diagnosis of both symptomatic and asymptomatic gonococcal infections, which is critical to the control of the disease [24]. Second, specimens collected for NAATs do not require the organism to be viable for detection and so require less stringent transport conditions compared with those collected for bacterial culture. Finally, NAATs can be used effectively on noninvasive specimens such as urine and self-collected specimens [6, 24 ]. N. gonorrhea NAATs do have some limitations; these include the typical problems associated with the use of NAAT protocols, such as high cost, carryover contamination, inhibition of the reaction, high quality control requirements, and the absence of antibiotic resistance data [25]. In addition, DNA amplification tests may not be suitable for test-of-cure because gonococcal DNA may be present for weeks after successful treatment of an infection [25].

Recommendation: The Centers for Disease Control and Prevention (CDC,USA) now promotes nucleic acid amplification testing, also known as NAAT, as the preferred method to detect these STDs in men and women in its latest recommendations to 
clinical laboratories [26]. The NAAT method detects the DNA genes of the bacteria causing the infection. It does this in part by amplifying or making numerous copies of the genetic material so that the detection system can identify the presence of the bacteria. It is theoretically able to detect as little as a single copy of bacterial nucleic acid in a sample taken from the individual tested [26].

\section{Antimicrobial treatment}

The widespread distribution of $N$. gonorrhea strains which are resistant to commonly and previously used antibiotics is a significant global public health problem, and it is a major threat to reducing the impact of STIs worldwide [27-31]. Recent studies from different continents reported increased antibiotic resistance to most previously used drugs such as penicillin, tetracycline and fluoroquinolones [3134]. Resistance to ciprofloxacin has reached $52.9 \%$ in Europe, $74.5 \%$ in Russia, $86.5 \%$ in the USA and $93.8 \%$ in China [30-31]. Moreover, a meta-analysis conducted in China and a report from the French National Reference Centre (CNR) have described an important resistance rate to tetracycline $(82.4 \%$ and $86 \%$, respectively) [32].

Few studies have recently reported on antibiotic resistance among $N$. gonorrhea isolates in Arab countries $[35,36]$. A study carried by Hamze et al., 2016 [35], indicated that a significant increase in the prevalence of gonorrhea in Tripoli/ North of Lebanon, a city suffering from a decline in the current socioeconomic situation with a high unemployment rate, extreme poverty and the influx of thousands of Syrian refugees. All their s $N$. gonorrhea trains were isolated from male patients. The study demonstrated $N$. gonorrhea strains showed significant rates of resistance to quinolone, macrolide and tetracycline drugs, with a resistance prevalence rates of $72.3 \%$ to nalidixic acid, $40.4 \%$ to ofloxacin, $38.3 \%$ to ciprofloxacin, $40.4 \%$ to azithromycin, $23.4 \%$ to tetracycline and $21.3 \%$ to minocycline [35]. While a recent study from Morocco reported that a to- tal of 72 isolates were examined, and a significant resistance to tetracycline (92.8\%) and ciprofloxacin (86.8\%), which were used as first-line treatment in gonococcal infections in Morocco. However, no resistance was found to spectinomycin, ceftriaxone or cefixime in all the isolates [36].

Currently, the treatment of gonococcal infections in many countries is limited to the use of thirdgeneration cephalosporins such as ceftriaxone and cefixime [22-26]. The results of a recent study showed that the gonococcal genotypes persisting in the population and fluctuated significantly within a 3 year period, with numerous other genotypes appearing or disappearing. Significant fluctuations in the most common genotypes accounted for the majority of observed increases in both ciprofloxacin and penicillin resistance. Single-year genotypes contributed to $\sim 20 \%$ of ciprofloxacin and penicillin resistance in each year. The study assumed that these changes determines $N$. gonorrhea antimicrobial resistance levels within the population [27].

It is important to note that some patients with gonococcal infection should be treated for possible C. trachomatis coinfection if chlamydia has not been ruled out [11]. For infections acquired via consensual sexual activity, patients should be advised to be abstinent for at least one week after treatment to prevent reinfection. Patients should also be advised to contact their sexual partners and to encourage their treatment. This strategy greatly reduces the public health burden of gonococcal disease [11].

The epidemiology of antimicrobial resistance in N.gonorrhea has changed decisions about gonococcal treatment recommendations because of shifts in antimicrobial resistance patterns in many countries over the world. The emergence of fluoroquinoloneresistant $N$. gonorrhea in the United States prompted the CDC in 2007, to stop recommending fluoroquinolones for treatment of gonorrhea, leaving cephalosporins as the only remaining class of antimicrobials available for treatment of gonorrhea in the United States [36]. Criteria for resistance to cefixime and cef- 
triaxone have not been defined by the Clinical and Laboratory Standards Institute (CLSI). However, isolates with cefixime or ceftriaxone MICs $\geq 0.5 \mu \mathrm{g} / \mathrm{mL}$ are considered to have decreased susceptibility [37]. Because of the prevalence of tetracycline resistance among N. gonorrhea isolates worldwide, particularly those with elevated cefixime MICs, the use of azithromycin as the second antimicrobial is preferred. However, decreased susceptibility of $N$. gonorrhea to cephalosporins and other antimicrobials is expected to continue; local and country surveillance for antimicrobial resistance is essential for guiding therapy recommendations [38].

\section{Recommendation}

The new CDC guidelines recommend either the association of a cephalosporin with azithromycin or a higher ceftriaxone dose. Gonococcal strains with elevated MICs to cefixime also are likely to be resistant to tetracyclines but susceptible to azithromycin in the United States. Consequently, only one regimen, dual treatment with ceftriaxone and azithromycin, is recommended for treatment of gonorrhea [39]. However, in the case of azithromycin allergy, doxycycline (100 mg orally twice a day for 7 days) can be used in place of azithromycin as an alternative second antimicrobial drug when used in combination with ceftriaxone or cefixime [39].

In conclusion, gonorrhea infection is still highly important public health issue and should be controlled by rapid diagnostic methods and treated without any delay with effective antimicrobial drugs.

\section{References}

1. World Health organization. Sexually transmitted infections (STIS), Fact sheet, updated August 2016 ( WHO reference number: WHO/RHR/16.09).WHO, Geneva, Switzerland.

2. Newman L, Rowley J, Vander Hoorn S, Wijesooriya NS, Unemo $\mathrm{M}$, et al. (2015) Global Estimates of the Prevalence and Incidence of Four Curable Sexually Transmitted Infections in 2012 Based on Systematic Review and Global Reporting. PLoS ONE 2015; 10:12.

3. Centers for Disease Control and Prevention. Sexually Transmitted Disease Surveillance, 2015. Atlanta, GA: US Department of
Health and Human Services; October 2016

4. Memish ZA, Filemban SM, Al-Hakeem RF, Hassan MH, AlTawfiq JA. Sexually transmitted infections case notification rates in the Kingdom of Saudi Arabia, 2005-2012. J Infect Dev Ctries 2016; 10(8):884-887.

15. Gdoura R, Kchaou W, Ammar-Keskes L, Chakroun N, Sellemi A, et al. Assessment of Chlamydia trachomatis, Ureaplasma urealyticum, Ureaplasma parvum, Mycoplasma hominis, and Mycoplasma genitalium in semen and first void urine specimens of asymptomatic male partners of infertile couples. J Androl 2008; 29(2): 198- 206.

6. Abusarah EA, Awwad ZM, Charvalos E, Shehabi AA. Molecular Detection of potential sexually transmitted pathogens in semen and urine specimens of infertile and fertile males. Diagn Microbiol Infect Dis 2013;77(4): 283-286.

7. Gaydos CA, Cartwright CP, Colaninno P, Welsch J, Holden J, et al. "Performance of the Abbott RealTime CT/NG for detection of Chlamydia trachomatis and Neisseria gonorrhoeae," J Clinic Microbiol 2010; 48: 9, pp. 3236-3243.

8. Miller KE. Diagnosis and treatment of Neisseria gonorrhoeae infections. Am. Fam. Physician 2006; 73(10):1779-84

9. Huppert JS, Biro F, Lan D, Mortensen JE, Reed J, Slap GB.Urinary symptoms in adolescent females: STI or UTI? J Adolesc Health 2007; 40:418.

10. Da Ros CT, Schmitt Cda S. Global epidemiology of sexually transmitted diseases. Asian J Androl 2008; 10(1):110-4.

11. Karnath BM. Manifestations of Gonorrhea and Chlamydial Infection. Hosp Physician 2009; 45(4):44-48.

12. Holder NA. Gonococcal infections. Pediatr Rev 2008; 29(7):228-34.

13. Pellati D, Mylonakis I, Bertoloni G, Fiore C, Andrisani A. et al. Genital tract infections and infertility. Eur J Obstet Gynecol Reprod Biol 2008;140(1):3-11.

14. Liu B, Roberts CL, Clarke M, et al. Chlamydia and gonorrhoea infections and the risk of adverse obstetric outcomes: a retrospective cohort study. Sex Transm Infect 2013; 89:672.

15. Krause W. Male accessory gland infection. Andrologia 2008;40(2):113-6.

16. Ochsendorf FR. Sexually transmitted infections: impact on male fertility. Andrologia 2008; 40(2):72-5.

17. Day AC, Ramkissoon YD, George S, and Corbett MC. Don't forget Gonococcus! Eye (Lond) 2006; 20(12):1400-2.

18. Ochsendorf FR. Sexually transmitted infections: impact on male fertility. Andrologia 2008; 40(2):72-5.

19. Janda WJ, Knapp JS. Neisseria and Moraxella catarrhalis. In: Murray PR, Baron EJ, Pfaller MA, Jorgensen JH, Yolken RH, eds. Manual of Clinical Microbiology, 8th edn. Washington: American Society Microbiology, 2003:585-608

20. Hansen L, Wong $T$, Perrin M. Gonorrhoea resurgence in Canada. Int J STD AIDS 2003;14:727-31.

21. Dillon JR, Carballo M, Pauzé M. Evaluation of eight methods for identification of pathogenic Neisseria species: NeisseriaKwik, RIM-N, Gonobio-Test, Minitek, Gonochek II, GonoGen, Phadebact Monoclonal GC OMNI Test, and Syva MicroTrak Test. J Clin Microbiol 1988;26:493-7.

22. Tabrizi SN, M. Unemo AE, Limnios TR, Hogan SO, Hjelmevoll SM, et al.. Evaluation of Six Commercial Nucleic Acid 
Amplification Tests for Detection of Neisseria gonorrhoeae and Other Neisseria Species. J Clin Microbiol 2011; 49:3610-3615.

23. Schweitzer VA, van Dam AP, Hananta IP, Schuurman R, Kusters $J G$, Rentenaar RJ. Identification of Neisseria gonorrhoeae by the Bruker Biotyper MatrixAssisted Laser Desorption IonizationTime of Flight Mass Spectrometry System Is Improved by a Database Extension. J Clin Microbiol 2016; 54: 4, 1130-1132.

24. Trembizki E, Costa AM, Tabrizi SN, Whiley DM, Twin J. Opportunities and pitfalls of molecular testing for detecting sexually transmitted pathogens. Pathology 2015; 47(3):219-26.

25. Whiley DM, Tapsall, JW, Sloots, TP. Nucleic acid amplification testing for Neisseria gonorrhoeae: an ongoing challenge. J Mol Diagn 2006; 8(1):3-15.

26. Http://www.cdc.gov/mmwr/preview/mmwrhtml/rr6302a1.htm through http://www.cdc.gov. Accessed May 2014.

27. Lahra MM, Trembizki E, Buckley C, Donovan B, Chen M, et al. GRAND Study Investigators. Changes in the rates of Neisseria gonorrhoeae antimicrobial resistance are primarily driven by dynamic fluctuations in common gonococcal genotypes. J Antimicrob Chemother 2017;1;72(3):705-711

28. Kubanov A, Vorobyev D, Chestkov A, Leinsoo A, Shaskolskiy $B$, et al. Molecular epidemiology of drug-resistant Neisseria gonorrhoeae in Russia (Current Status, 2015). BMC Infect Dis $2016 ; 16: 389$.

29. Chen SC, Yin YP, Dai XQ, Unemo M, Chen XS.First nationwide study regarding ceftriaxone resistance and molecular epidemiology of Neisseria gonorrhoeae in China. J Antimicrob Chemother $2016 ; 71(1): 92-9$.

30. Lee H, Unemo M, Kim HJ, Seo Y, Lee K, Chong Y. Emergence of decreased susceptibility and resistance to extended-spectrum cephalosporins in Neisseria gonorrhoeae in Korea. J Antimicrob Chemother. $2015 ; 70(9): 2536-42$

31. Kirkcaldy RD, Kidd S, Weinstock HS, Papp JR, Bolan GA. Trends in antimicrobial resistance in Neisseria gonorrhoeae in the USA: the Gonococcal Isolate Surveillance Project (GISP), January 2006-June 2012. Sex Transm Infect $2013 ; 89$ 4:iv5-10

32. Chen Y, Gong Y, Yang T, Song X, Li J, et al. Antimicrobial resistance in Neisseria gonorrhoeae in China: a meta-analysis. BMC Infect Dis 2016;16: 108.

33. Pond MJ, Hall CL, Miari VF, Cole M, Laing KG, Jagatia $H$, et al. Accurate detection of Neisseria gonorrhoeae ciprofloxacin susceptibility directly from genital andextragenital clinical samples: towards genotype-guided antimicrobial therapy. J Antimicrob Chemother 2016;71:897-902.

34. Goubard A, Sednaoui P, Cambau E, Bercot B. Rapport d'activite 2013 du CNR des gonocoques. Paris, France: Institut Alfred Fournier/Groupe Hospitalier Saint Louis-Lariboisiere-Fernand Vidal; 2014 . http://www.institutfournier.org.

35. Hamze M, Osman M, Achka M, Mallat H, Dabboussi F. Alarming increase in prevalence of Neisseria gonorrhoeae infections associated with a high level of antibiotic resistance in Tripoli, Lebanon. I J Antimicrob Agents. Published on line: September 7, 2016; P576-577.

36. Hançali A, Ndowa F, Bellaji B, Bennani A, Kettani A, Charof $R, E l$ Aouad R. Antimicrobial resistance monitoring in Neisseria gonorrhoeae and strategic use of funds from the Global Fund to set up a systematic Moroccan gonococcal antimicrobial surveillance programme. Sex Transm Infect 2013; 4:iv24-7

37. National Committee for Clinical Laboratory Standards. Approved Standard M100-S20 performance standards for antimicrobial susceptibility testing: twentieth informational supplement. Wayne, PA: Clinical and Laboratory Standards Institute; 2010

38. Update to CDC's sexually transmitted diseases treatment guidelines, 2006: fluoroquinolones no longer recommended for treatment of gonococcal infections. MMWR Morbid Mortal Wkly Rep 2007; 56:332-6.

39. Neisseria gonorrhoeae Antimicrobial Susceptibility Surveillance - The Gonococcal Isolate Surveillance Project, 27 Sites, United States, 2014. MMWR July 14, 2016.

\section{Comment on this article:}

\section{f] $[B$ in $S$}

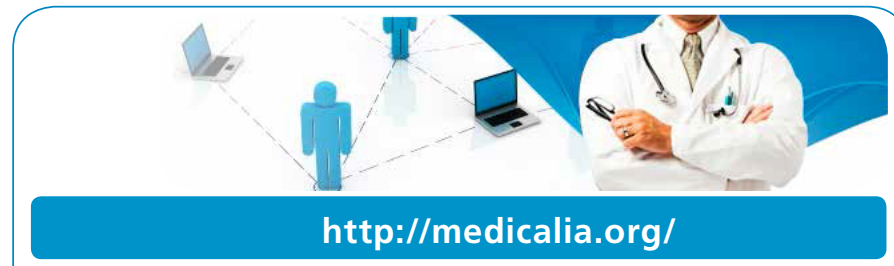

Where Doctors exchange clinical experiences, review their cases and share clinical knowledge. You can also access lots of medical publications for free. Join Now!

\section{Publish with iMedPub}

http://www.imed.pub

The Journal is an open access peer-reviewed journal that publishes scientific papers about all aspects of antimicrobials. The journal will publish original research articles, reviews, brief reports and case reports dealing with basic and clinical antibacterial agents, antiviral, antiprotozoals, antituberculuous, antifungal and antihelminthes agents.

All manuscripts must be prepared in English, and are subject to a rigorous and fair peer-review process. Accepted papers will immediately appear online.

The journal aims to advance the knowledge, attitude and the research of chemotherapy in the Arabic world in cooperation with international, national scientific and public societies as well as research centers with similar aims and objectives. 